Journal: Expert Opinion on Biological Therapy

Title: Gene Therapy for Inherited Retinal and Optic Nerve Degenerations

Nicholas A. Moore, Nuria Morral, Thomas A. Ciulla \& Peter Bracha

KEY WORDS: gene therapy; adeno-associated virus; inherited retinal disease; Leber's Congenital Amaurosis; retinitis pigmentosa; choroideremia; Stargardt Disease

WORD COUNT (excluding abstract and references): 7,137 words

\begin{abstract}
:
INTRODUCTION:

The eye is a target for investigational gene therapy due to the monogenic nature of many inherited retinal and optic nerve degenerations (IRD), its accessibility, tight blood-ocular barrier, the ability to non-invasively monitor for functional and anatomic outcomes, as well as its relative immune privileged state.
\end{abstract}

\begin{abstract}
AREAS COVERED:
Vectors currently used in IRD clinical trials include adeno-associated virus (AAV), small single-stranded DNA viruses, and lentivirus, RNA viruses of the retrovirus family. Both can transduce non-dividing cells, but AAV are non-integrating, while lentivirus integrate into the host cell genome, and have a larger transgene capacity. This review covers Leber's congenital amaurosis, choroideremia, retinitis pigmentosa, Usher syndrome, Stargardt disease, Leber’s hereditary optic neuropathy, Achromatopsia, and X-linked retinoschisis.

\section{EXPERT OPINION:}

Despite great potential, gene therapy for IRD raises many questions, including the potential for less invasive intravitreal versus subretinal delivery, efficacy, safety, and longevity of response, and acceptance of novel study endpoints by patients, clinicians, and payers. Also, ultimate adoption of gene therapy for IRD will require widespread genetic screening to identify and diagnose patients based on genotype instead of phenotype, as well as new reimbursement models for one-time therapies.
\end{abstract}

This is the author's manuscript of the article published in final edited form as:

Moore, N. A., Bracha, P., Hussain, R. M., Morral, N., \& Ciulla, T. A. (2017). Gene therapy for age-related macular degeneration. Expert Opinion on Biological Therapy, 17(10), 1235-1244. https://doi.org/10.1080/14712598.2017.1356817 


\section{INTRODUCTION:}

\subsection{Overview}

Inherited retinal degenerations (IRD) are a rare and heterogenous group of diseases that collectively result in progressive retinal degeneration with resultant severe visual impairment; over 200 causative mutations have been identified. The most common IRDs include Leber's congential amaurosis (LCA), choroideremia, retinitis pigmentosa (RP), Usher syndrome, Stargardt disease, Leber's hereditary optic neuropathy (LHON), Achromatopsia, and X-linked retinoschisis (XLRS) (1). The blinding nature of these conditions and lack of effective treatment highlight the need for therapeutic innovation. The fact that the disease-causing genes have been identified, together with their monogenic etiology facilitates the potential for gene therapy. Furthermore, IRDs also attracted interest because both the United States Food and Drug Administration (FDA) and European Medicines Agency (EMA) have incentivized development of therapies for rare diseases. In the US, the Orphan Drug Act of 1983 defined orphan diseases as those that affected fewer than 200,000 Americans. The Orphan Drug Act provided sponsors with 7 years of exclusivity, tax credits to defray the cost of development, waived FDA fees, and provided protocol assistance. The EMA provided similar incentives.

\subsection{Introduction to viral vectors}

For a disease to be a good candidate for treatment using gene therapy, several factors need to be fulfilled: (i) Substantial disease burden, and favorable risk/benefit ratio compared to alternative therapy; (ii) The relevant gene/locus has been identified, and there is sufficient knowledge of the disease process; (iii) The appropriate target cell(s) can be targeted with a gene delivery method; (iv) Phenotype improvement is attained with low expression levels of the gene, and its overexpression is not associated with toxicity. Recessive single gene disorders are the most amenable to be treated by gene therapy, because the mutations causing the disease generally lead to loss-of-function of the gene product and therefore, total absence or near absence of functional protein. Gene replacement therapy in these cases can correct the lost function by delivery of the normal gene. Furthermore, in some instances restoring a small percentage of the normal level of the gene product is sufficient to revert the phenotype. The majority of gene therapies to treat inherited ocular diseases target autosomal recessive diseases. For example, LCA, 
type 2, mediated by mutations in the RPE65 gene is a recessive monogenic disorder in which gene-based therapy corrects the lost function. In contrast, gain-of-function mutations produce dominant phenotypes. These diseases may be less amenable to be treated by gene therapy, as one gene copy expresses an abnormal product that has to be suppressed, while the other expresses a protein that functions normally (2). Consequently, developing a gene therapy for autosomal dominant RP has been more challenging.

A variety of viral and non-viral gene delivery methods have been developed over the past couple of decades. Choosing one delivery method over another is influenced by the tissue to be targeted, the cloning capacity of the vector (which determines the size of the expression cassette that can be accommodated in the genome of the virus), and safety concerns (inflammatory responses, and possibility of genotoxicity/insertional oncogenesis). Adeno-associated virus (AAV) and lentivirus vectors have been used in the treatment of retinal inherited diseases. Adeno-associated viruses are small, singlestranded DNA viruses of the parvovirus family (3). AAV vectors transduce quiescent tissues, and their genome is mainly maintained as extrachromosomal monomeric and concatemeric circles (4). Over 100 different AAV serotypes have been described, each of them displaying enhanced tropism for a specific set of tissues. To date, AAV is the most common viral vector utilized in retinal genetic therapy. AAV serotypes 2, 5, and 7-9 are capable of transducing photoreceptors, while virtually every AAV serotype is capable of infecting the retinal pigment epithelium (RPE) (5). Multiple features make it an excellent vector choice for these diseases, including the non-integrating nature, low inflammatory potential, low immunogenicity, low retinal toxicity at appropriate doses, non-pathogenic nature, ability to transduce non-dividing cells, and excellent track record of safety in human trials (6-8). AAV vectors do have limitations, which include having a restricted transgene capacity (4.5-5.0 kb) and the risk of being rapidly eliminated by the humoral immune response in patients who have previously been exposed to the virus (7). However, the risk for immunogenicity with AAV vectors is low when targeting relatively immune-privileged tissues such as the retina (8). Transduction efficiency using AAV is hindered by the requirement of converting the single-stranded DNA genome to doublestranded DNA, a step that typically takes weeks. To overcome this limitation, investigators have developed self-complementary AAV vectors (scAAV), which overcome the need for dsDNA conversion by packaging a dimeric inverted repeat genome that folds into double stranded DNA (9-10). The caveat to this approach is that the size of the expression cassette has to be reduced by half, which is a constraint to the already limited cloning capacity of AAV.

Lentiviruses are RNA viruses of the retrovirus family. Commonly used lentiviral vectors derive from the human immunodeficiency virus 1 (HIV1) or the equine infectious anemia virus (EIAV) (11). Lentivirus vectors efficiently integrate their genome into the host cell genome, and do not require cell division for integration (12). Additionally, lentiviruses are able to transduce both dividing and non-dividing cells. An added attractive feature is the cloning capacity, up to $10 \mathrm{~kb}$ (13). Lentiviruses have recently become a popular choice because they have improved safety features relative to gamma-retroviruses, as the vector genome does not preferentially integrate in the proximity of oncogenes (14). 


\subsection{Methods of introducing viral vectors to the eye}

The eye provides an excellent model for investigating gene therapy because the ocular relative immune-privilege limits an immune response to the implanted genetic material and the tight blood-ocular barrier limits the systemic dissemination of the introduced genetic material. Additional advantages include ease of accessibility for delivery of the genetic material directly to the target cells of interest, the non-invasive ability to monitor for disease progression and response to therapy, and use of the contralateral eye as an in vivo control (15).

Vector delivery to the target retinal tissue involves two potential methods. The most commonly investigated method involves pars plana vitrectomy (PPV) followed by retinotomy and injection of the viral vector with genetic material into the subretinal space (Figure 1). This more invasive method creates a temporary retinal detachment, but allows for direct delivery to the cells of interest. The virus then "infects" the RPE cells or photoreceptors, causing the host cells own translational machinery to express the protein. Alternatively, injection of the vector into the vitreous cavity has been attempted, and although this method may be less invasive and potentially have fewer procedure-related complications, the penetration of viral vector to the target tissue is perceived to be inferior to that of subretinal injections (16) (Figure 2). Additionally, animal models have demonstrated an induced humoral immune response to intravitreally delivered vectors that was not observed with subretinally delivered vectors (17). This review will explore the current state of genetic replacement therapy research and clinical trials as it pertains to a subset of IRDs with specific genetic mutations including Leber's congential amaurosis (LCA), choroideremia, X-linked and autosomal recessive retinitis pigmentosa (RP), Usher syndrome, Stargardt's disease, Leber's hereditary optic neuropathy (LHON), Achromatopsia, and X-linked retinoschisis (XLRS) (Table 1 and 2).

\subsection{Gene therapies for Leber's Congenital Amaurosis:}

Leber's congenital amaurosis (LCA) is a heterogenous group of infantile-onset, retinal dystrophy caused by a variety of genetic mutations (18-19). It is a rare, visually devastating disease with an estimated prevalence of 1 in 50,000-100,000 resulting in nystagmus and severely decreased visual acuity (VA) in early infancy; complete blindness typically ensues by the third to fourth decade of life (20). One of the pathologic genetic mutations of interest involves the RPE65 gene encoding retinoid isomerohydrolase, a $65-\mathrm{kD}$ protein expressed in the RPE, which plays a key role in the visual cycle. When light strikes rhodopsin (composed of the protein opsin bound to the chromophore 11-cis-retinal) in the rod outer segments, 11-cis-retinal is converted to its trans isomer. This, in turn, activates the opsin and initiates a signal transduction cascade, closing a cyclic GMP-gated cation channel, and hyperpolarizing the photoreceptor cell. In the visual cycle, 11-cis retinal must ultimately be converted back from its trans isomer, via a series of enzymes, including Lecithin Retinol Acyltransferase (LRAT) and retinoid isomerohydrolase (encoded by the RPE65 gene), both of which are located in the RPE (21-24). Deficiency in either of these enzymes leads to early age onset progressive degeneration of rod photoreceptors and ultimately results in irreversible loss of cone- 
mediated vision, a degenerative process that may be mitigated by early initiation of gene therapy.

Mutations of RPE65, which encodes retinoid isomerohydrolase, result in retinitis pigmentosa 20 (RP20) and LCA, type 2, a subtype of LCA that has been extensively evaluated in preclinical animal models. Gene-replacement therapy, through subretinal delivery of recombinant AAV vectors containing RPE65 cDNA, has been shown to improve electrophysiological responses and functional vision in the Swedish Briard dog, a naturally occurring animal model with mutated RPE65 (25-26). The success of the preclinical murine and large-animal models translated into a number of human trials evaluating the safety of injecting AAV vectors containing the human RPE65 coding sequence (26). All of the studies reviewed below, have similar outcomes, but vary slightly in their designs with respect to the use of specific promoters for enhancing the expression of RPE65, the volume of vector injected, and the surgical protocol for vector delivery.

Three small phase I studies were published in 2008 and are summarized in Table 1 (2729). In these studies, adverse events were related to the surgical procedure and there were preliminary signals of efficacy. Major similarities in methods between studies included the utilization of a PPV with subretinal vector delivery. The favorable safety profile and possible effectiveness of these pilot studies led Maguire et al. to perform a doseescalation phase I study of 12 patients age 8-44 years (NCT00516477) (30). Patients were randomized to receive a low dose $\left(1.5 \times 10^{10}\right.$ vector genomes $(\mathrm{vg})$ in $\left.0.15 \mathrm{~mL}\right)$, a medium dose $\left(4.8 \times 10^{10} \mathrm{vg}\right.$ in $\left.0.15 \mathrm{~mL}\right)$, or a high dose $\left(1.5 \times 10^{11} \mathrm{vg}\right.$ in $\left.0.3 \mathrm{~mL}\right)$ subretinally following PPV. They reported AAV2-hRPE65v2 vector was well tolerated, and all patients showed sustained improvement in subjective and objective measurements, including pupillometry, VA, visual field testing, nystagmus, and ambulatory behavior. (30). The same group reported that visual function improvements remained stable up to 3 years (31). Alternatively, Jacobson et al. and Bainbridge et al. reported less promising long-term outcomes; direct comparison between these groups is difficult, however, as they used different virus vectors, manufacturing processes, and lower doses (32-33).

Given the favorable results from the phase 1 trial reported by Maguire et al., Spark therapeutics is sponsoring commercialization of this therapy in their lead ocular program. The evaluation of RPE65 gene (AAV2-hRPE65v2) administered to the contralateral eye in patients enrolled in the phase 1 study was recently released (NCT01208389) (34). Since there were no dose-limiting toxicities in the first study, the highest dose of $1.5 \times 10^{11}$ vg in $0.3 \mathrm{~mL}$ was subretinally injected into the contralateral, previously uninjected, eyes in 11 of the 12 patients. Although there was theoretical concern surrounding the possible increased risk of an immune response due to the previously introduced AAV2 vector, most patients did not experience significant immunogenicity. Obstacles for evaluating visual function with VA testing after genetic treatment may be limited by developmental deficiencies in the visual cortex in individuals with poor vision since birth. In addition to amblyopia, other obstacles include limited dynamic range of VA testing, and the fact that LCA and RP are predominantly rod-mediated disorders with secondary effects on cone function and VA, decreasing its sensitivity to change in visual function. To more fully 
assess the functional benefits of RPE65 gene therapy in LCA patients, who do not perform well on traditional methods of vision testing, a proprietary multiluminace mobility test (MLMT) was developed, with feedback from the FDA in order to functionally assess VA, visual fields, light sensitivity, and mobility. In this test, patients progress through an "obstacle course", with 12 standardized templates, under multiple standardized lighting conditions, to determine the lowest illumination under which they can successfully navigate the course in under 3 minutes. The seven levels of illumination reproduce the lighting conditions encountered in daily life, from 1 lux, simulating a moonless night, to 400 lux, simulating an office setting. A change in score, based on illumination level, functions as the endpoint and has been validated. Compared with baseline, of ten participants analyzed, improvements in MLMT and full-field light sensitivity testing (FST) in the injected eye by day 30 persisted to year 3 (MLMT $\mathrm{p}=0.0003$, FST $\mathrm{p}<0.0001$ ), and no significant change was seen in the previously injected eyes or VA assessments over the same time period (34).

According to publicly disclosed information, Spark initiated a Phase III trial in November 2012 enrolling 31 patients with a mean age of 15 years; the patients were randomized 2:1 into control or receiving $1.5 \times 10^{11} \mathrm{vg}$ of SPK-RPE65 in both eyes within 18 days. After the 12-month time point, the control subjects were eligible to cross over into the treatment group. In October 2015, Spark announced positive top-line results. There were no serious adverse events (SAEs) or significant immune responses related to SPKRPE65. The trial met its primary endpoint of change at one year in the bilateral MLMT from baseline $(\mathrm{p}=0.001)$, with an average improvement 1.8 light levels in the treatment group versus 0.2 light levels in the control group. Of the 20 treated subjects, 13 (65\%) were able to pass the MLMT at the lowest light level (1 lux, demonstrating maximal possible improvement) at one year versus none in the control group. Secondary endpoints successfully met included FST with 100-fold improvement in light sensitivity in treated subjects $(\mathrm{p}<0.001)$ and MLMT change score for the first injected eye $(p=0.001)$. Although the VA secondary endpoint did not reach statistical significance, a beneficial trend was noted among treated patients (35). In May 2017, Spark announced that it had completed its rolling Biologics License Application (BLA) submission to the FDA for SPK-RPE65, Voretigene Neparvovec, which could potentially represent the first approved genetic therapy in the United States (36).

In addition, alternative vectors such as AAV4-RPE65 have been evaluated for RPE65 specific retinal dystrophies (NCT01496040) (37). Other companies that are sponsoring gene therapy trials for LCA include Applied Genetics Technology Corporation (AGTC) (38) and MeiraGTx. MeiraGTx has initiated a phase I/II clinical trial of AAVOPTRPE65 (39).

In addition to RPE65, a mutation in GUCY2D has been implicated in LCA type 1. GUCY2D encodes retinal guanylyl cyclase-1, a protein expressed in the outer photoreceptors that responds to low intracellular calcium levels and produces cGMP to return the photoreceptors to the pre-excitation state after light exposure (40-41). A mutation in GUCY2D caused decreased VA and ERG dysfunction, but unlike LCA2 patients often have normal laminar structures of the retina (40). A mouse model was 
recently rescued with an AAV5-GRK1-GUCY2D delivered gene replacement and the results found a restoration of retinal function for 6 months (40). There are no clinical trials to date for GUCY2D mutations, but these animal model findings indicate that patients with this mutation may benefit from future gene therapy.

Finally, the retinitis pigmentosa GTPase regulator protein (RPGRIP1) gene encodes a structural protein that localizes to the connecting cilium linking the inner to the outer segment and is involved in the regulation of protein trafficking across the cilium (19). Loss of this gene has been implicated in photoreceptor degeneration and LCA development. The RPGRIP $1^{-/-}$knockout mouse develops a severe retinal degeneration. AAV2 constructs with an opsin promoter and murine RGRIP1 cDNA were injected subretinally into the knockout mouse model. Treated eyes were found to have photoreceptor structural preservation as determined by a thicker and well-developed outer nuclear layer on histologic examination. Additionally, the RPGRIP restored normal subcellular localization of the RPGR. ERG b-waves declined by 22\% per month in the untreated eyes, while the treated eyes declined by $6 \%$ per month. Five months post injection, the mean b-wave amplitudes in the treated eyes were significantly higher than the b-wave amplitudes in control eyes. (42)

\subsection{Gene therapies for Choroideremia:}

Choroideremia is an X-linked chorioretinal dystrophy characterized by diffuse, progressive degeneration of the RPE, photoreceptors and choriocapillaris. It has a prevalence of about 1 in 50,000 with northern Finland having the highest reported rate (43). The disease is characterized by progressive vision loss, but unlike LCA, central vision is maintained until the fourth or fifth decade of life and males are predominantly affected due to its X-linked inheritance pattern. It is caused by a mutation in the CHM gene, which encodes for Rab escort protein-1 (REP-1) and is the focus of ongoing gene replacement therapy. REP-1 is a ubiquitously expressed protein required for the efficient geranylgeranylation prenylation, (a post-translational modification of proteins involving the attachment of geranylgeranyl isoprene units) of ras-related GTPases, or Rab proteins, which are integral to the trafficking of vesicles in endocytic and exocytic pathways (4446). It has been suggested that this lack of protein affects opsin transport to photoreceptor outer segments, apical migration of RPE melanosomes, and the phagocytosis of photoreceptor outer segments by the RPE resulting in progressive RPE and photoreceptor cell death (47).

Recent success in the treatment of LCA, as reviewed above, has brought about the evaluation of AAV vector-mediated gene replacement in choroideremia. The cloning capacity of AAV vectors is approximately 4.5-5.0 kb, which is greater than the approximately $1.9 \mathrm{~kb}$ coding sequence of REP-1, making it an excellent vector for delivery (48-50). The replacement of the REP-1 gene was evaluated in a choroideremia murine model, in which mice were designed to have an REP-1 deficiency. In this preclinical model, REP-1 replacement via the subretinally injected AAV2 vector was demonstrated to be feasible, safe, and improved ERG responses (51). 
This proof-of-concept study led to a human multicenter phase $1 / 2$ clinical trial, in which six male patients (aged 35-63 years) were administered with AAV.REP1 (NCT01461213). The surgical technique involved PPV followed by placement of $0.1 \mathrm{~mL}$ of $1 \times 10^{10}$ AAV2.REP1 genome particles into the subretinal space in 5 of 6 patients. In the sixth patient, a reduced dose of $0.1 \mathrm{~mL}$ of $6 \times 10^{9}$ was injected. The sixth patient was injected with a smaller dose of viral particles because of difficulty in creating a retinal bleb and concern about stretching the papillomacular bundle. VA was measured using the Early Treatment Diabetic Retinopathy Study (ETDRS) letters, which requires a change of at least 0.2 logMAR (2 lines of letters) to distinguish from no change (52). At six months, the mean ETDRS letters was +3.8 letters in the treated eyes in comparison to +1.5 letters in the control eyes, although statistical analysis was not published due to the small sample size. Patient 1 had a +21 letter improvement and patient 4 had a +11 letter improvement, with the other patients having a marginal loss in VA. On microperimetry, a mean increase was noted in retinal sensitivity with respect to the dimmest stimulus seen $(2.3 \mathrm{~dB})$, with respect to mean retinal sensitivity $(1.7 \mathrm{~dB})$ and with respect to total number of test points seen (1.5), in contrast to a reduction in all of these parameters in the patient's contralateral eye. Mean retinal sensitivity increased by $2.5 \mathrm{~dB}$ in the five eyes administered with the full dose of vector, but fell by $2.3 \mathrm{~dB}$ in the eye administered with the reduced dose. There was no observed immune response to the injected viral particles. Two patients had mild distortion of central vision, which resolved by 6 months. All patients had sub-clinical progression of lens opacification, as expected following PPV. No serious systemic or ocular adverse events were reported. Importantly, no retinal thinning or loss of VA was observed despite the surgically induced retinal detachment, establishing a favorable safety profile for this gene transfer protocol targeting the fovea. Interestingly, one patient demonstrated a shift in the preferred retinal locus from the fovea to the area adjacent to the site of vector treatment (44).

At 3.5 years, the patients who had initial improvement in VA maintained that VA. Three other patients did not have a significant change in VA and one patient had a decline in vision. The patient who had a decline in vision was the patient injected with a lower total vector dose (53). Based on this work from the University of Oxford, NightstaRx is sponsoring additional clinical trials with NSR AAV-REP1, which is expected to enter Phase 3 pivotal testing, pending final discussions with the FDA (54).

Spark Therapeutics has also sponsored an open-label, dose-escalating Phase 1/2 trial designed to assess the safety and preliminary efficacy of subretinal administration of investigational SPK-7001 in choroideremia. The trial enrolled 2 dose cohorts of 5 patients with advanced disease and initial efficacy analysis is expected in 2017 (55).

The pathogenesis of choroideremia is complex and it is thought to affect RPE, photoreceptors, and choroid, but which retinal layer is affected most in this disease has yet to be determined (46). Some studies have demonstrated that both RPE and photoreceptors are affected in CHM and that an affected RPE accelerates photoreceptor degeneration, highlighting RPE as the primary layer affected in CHM (56). Other studies have suggested that rod cell death results in secondary cone mediated death and is the primary site of CHM pathology (57). However, REP-1 was also localized to cone 
photoreceptors, raising the question as to whether rods and cones need to be independently targeted with vector therapy (58). This has led to the investigation of other vectors such as AAV8 that are being explored because of the increased tropism for photoreceptors when compared with the AAV2 vector. Preclinical in vitro and in vivo murine studies demonstrated successful dose responsive expression of REP-1 in photoreceptors using an AAV8 vector (59-60). The AAV2 viral vector was designed to target RPE cells and it is hypothesized that the use of a viral vector such as AAV8 that targets both RPE and photoreceptors may increase therapeutic efficacy and decrease the dosage required. Further advancements in CHM research has been limited by this complex pathophysiology and existing animal models that do not replicate the human condition (46). With better understanding of the pathology of CHM, clinical research developing an accurate animal model is being investigated as a way to contribute to innovative therapeutic approaches (46).

\subsection{Gene therapies for Retinitis Pigmentosa (RP):}

Retinitis pigmentosa is a heterogenous hereditary disorder resulting in diffuse, progressive photoreceptor loss preferentially affecting rod photoreceptors with secondary loss of cones. Epidemiologic studies vary by population, but RP is the most frequent form of inherited retinal dystrophies with a prevalence of 1 in 4,000 cases worldwide (61). Patients classically present with nyctalopia early in the course of disease, progressive loss of peripheral vision and eventually develop central visual impairment. On fundus examination, patients have arteriolar narrowing, bone spicule-like pigmentary changes and waxy pallor of the optic disc. A mutation in RHO, which encodes rhodopsin, was the first one associated with the disease, but numerous other genetic mutations have subsequently been identified, including genes encoding for mer receptor tyrosine kinase (MERTK), usherin, as well as retinitis pigmentosa GTPase regulator (RPGR) and retinoid isomerohydrolase (encoded by the RPE65 gene). As described above, these latter two mutations overlap in LCA, demonstrating the limited utility of the original phenotypic diagnoses in an era in which genotyping becomes more widely adopted.

MERTK encodes a tyrosine kinase that is required for phagocytosis of photoreceptor outer segments by the RPE and is associated with a rare form of autosomal recessive RP (62-64). The genetic mutation and its resultant pathology has been extensively studied in preclinical models mainly because it is the gene mutation in Royal College of Surgeon (RCS) rats, a popular model for retinal dystrophies, including RP (64). Several studies successfully treated RCS rats with the AAV2 vector carrying the MERTK gene and further improved the therapy through use of the RPE-specific promoter, vitelliform macular dystrophy (VMD2) (65-67). A phase I clinical trial sponsored by Fowzan Alkuraya (NCT01482195) utilizing an AAV2 vector with an RPE-specific promoter (VMD2) driving MERTK was published in 2016. To date, six patients received a submacular injection of AAV2-VMD2-hMERTK. One eye of each patient received a nonfoveal, submacular injection of the viral vector, with the first two patients receiving a dose of $0.15 \mathrm{ml}\left(5.96 \times 10^{10} \mathrm{vg}\right)$ and the four other patients receiving $0.45 \mathrm{ml}(17.88 \times$ $10^{10} \mathrm{vg}$ ). Postoperatively, one patient retained the subretinal bleb for a much longer than expected period of time (>3 weeks) and experienced "vibratory movement of the object." It was not clear to the authors if this was related to the bleb and neurophthalmic 
evaluation did not reveal an ocular movement disorder. Of note, two patients demonstrated an increased AAV antibody serum titer following the injection, but neither patient was positive for rAAV vector genomes using PCR. The clinical implication of this rise, as in the previously discussed sections, is not entirely clear, but the authors suspected the antibody detection to be cross-reactivity as opposed to true systemic viral exposure. No systemic complications attributed to the vector were observed. Three patients displayed improved VA in the treated eye at follow-up visits. However, this improvement in VA declined to baseline 2 years post treatment in two of the patients and only one patient demonstrated a sustained improvement in VA from baseline of 20/200 to $20 / 80$ at 2 years (68).

The retinitis pigmentosa GTPase regulator (RPGR) gene is involved in 70\% of X-linked RP and up to $20 \%$ of all RP cases. RPGR encodes for a transporter protein in the cilium, connecting the inner and outer segments of photoreceptors and suspected to play a role in transporting phototransduction components across the connecting cilium. X-linked RP due to RPGR mutations carries a poor prognosis associated with an aggressive course. AAV subretinal delivery of RPGR-ORF15 in an RPGR null murine model was found to have appropriate localization of transgene expression to the connecting cilium with structural and functional photoreceptor rescue (69). Additionally, subretinal AAV 2/5 RPGR was injected into canine models and demonstrated preserved photoreceptor morphology and improved rod and cone function (70). In March of 2017, NightstaRx announced that it has initiated a Phase I/II clinical trial (NCT03116113) of subretinal injection of AAV-XLRPRG gene therapy for X-linked RP caused by RPRG mutation. The clinical trial is an open-label dose escalation study with planned enrollment of 24 patients (54). MeiraGTx is also developing a gene therapy to target the RPGR gene in photoreceptor cells, but has not initiated clinical trials (39).

Unlike autosomal recessive or X-linked RP, a challenge in treating dominant RP (a significant proportion of RP cases) relates to the need to inhibit a gene, which can result in untoward effects on cellular physiology due to a narrow window of gene expression required for proper cellular function. To address this, Farrar et al. reviewed the different methods that are being developed to supplement normal gene expression while at the same time inhibiting the expression of the mutated gene (71). Another hurdle is that rhodopsin (RHO)-linked autosomal dominant RP ( 25\% of autosomal dominant RP) is caused by over 150 different mutations in the RHO gene alone, and the time and cost of developing sequence-specific inhibitors for each mutation is not economically feasible (72). Finally, most previously studied viral vectors were designed to target RPE cells, with minimal tropism for photoreceptors. Several alternative viral vectors, including AAV2/5, AAV2/8 and AAV2/rh10 have been designed and found to be tropic to photoreceptors and to be effective for gene transfer in animal models. However, they have not been evaluated for safety and efficacy in humans to date (72-73).

\subsection{Gene therapies for Usher Syndrome:}

Usher syndrome is an autosomal recessive genetic disease caused by a number of genetic mutations. One of the most investigated genes is Myocin VIIA and mutation in this gene is characterized by retinitis pigmentosa and progressive hearing loss with possible 
vestibular dysfunction (74). The prevalence of Usher syndrome has been reported to range from 3.2 to 6.2 per 100,000 (75). Myocin VIIA (MYO7A) is expressed in cochlear hair cells of the inner ear as well as in retinal photoreceptors and the RPE, where it plays a role in multiple cellular processes, including endocytosis and opsin delivery (76). Although the amount of MYO7A in photoreceptors is lower than that in the RPE, photoreceptors are affected before RPE cells in patients with Usher1B, indicating that photoreceptors are important cells to target in this disease.

Due to the large size of the MYO7A cDNA, delivery methods other than AAV are being investigated. The ability of lentivirus-MYO7A to restore RPE abnormalities has been shown in the MYO7A knockout mouse after subretinal injection of the vector at birth (77). Subretinal injections of EIAV-CMV-MYO7A (UshStat) in the mouse model of USH1B decreased the amount of photoreceptor loss and restored the transducing translocation in photoreceptors (77). In 2009, Oxford Biomedica entered into collaboration with SanofiAventis to investigate the utilization of the lentivirus gene delivery system for treating ocular diseases such as Usher syndrome type 1B and Stargardt disease (78). Currently patients with USH1B are being recruited for a phase I/II trial to assess the safety and tolerability of subretinal EIAV-CMV-MYO7A.

In addition to lentivirus, other delivery methods are being investigated. Specifically, DNA-compacted nanoparticles, which are single DNA molecules compacted polyethylene glycol-substituted polylysine 30-mers (CK30PEG). These have a $20 \mathrm{~kb}$ packaging capacity, providing future possibilities for gene therapy, with particular utility in treatment of Usher 2A syndrome, associated with mutations in the Usherin gene - a $15.6 \mathrm{~kb}$ sized gene that prohibits packaging into any AAV vectors (79). The safety and utility of the nanoparticles has been demonstrated in the lung, with introduction of the cystic fibrosis transmembrane conductance regulator through an intranasal route (80). However, nanoparticles have shown some limitations hindering their widespread use in ocular gene therapy. It was found to have lower transduction efficiency per vector genome as compared to AAV. This would require higher vector amounts and repeated dosing schedules, which could be dangerous in subretinal injections for ocular disease (81).

\subsection{Gene therapies for Stargardt Disease:}

Stargardt disease is a juvenile inherited macular dystrophy caused by a mutation in the ATP binding cassette subfamily A member 4 (ABCA4) gene with an estimated prevalence of between 1 in 8,000 to 10,000 (82). Subtle symptoms typically present during childhood followed by progressive macular atrophy, which ultimately results in severe vision loss in young adults. ABCA4 is localized to the outer segments of photoreceptors and acts as a membrane transporter for the recycling of a chromophore during the visual cycle. Specifically, ABCA4 binds with high affinity and shuttles Nretinylidene-phosphatidylethanolamine trapped inside the photoreceptor disc across the membrane surface, where it is ultimately further processed by the adjacent RPE cells as part of the visual cycle. Loss of ABCA4 function is associated with accumulation of toxic $N$-retinylidene- $N$-retinylethanolamine (A2E) in RPE cells, followed by severe RPE and macular photoreceptor death (83-84). The accumulation of this lipofuscin-like material results in the classical dark choroid found on fluorescein angiography. The 
ABCA4 gene $(6.8 \mathrm{~kb})$ exceeds the 4.5-5.0 kb capacity of the AAV vector and has required utilization of vectors such as the equine infectious anemia lentivirus (EIAV) for gene transfer (85). Subretinal injection of EIAV-ABCA4 was found to be effective in a knock out mouse model. Currently, Oxford Biomedica in coordination with Sanofi, is sponsoring an escalating dose Phase I/II EIAV-ABCA4 clinical trial investigating its utility in Stargardt disease (86-88).

\subsection{Gene therapies for Leber's Hereditary Optic Neuropathy (LHON):}

LHON is an inherited mitochondrial optic neuropathy with progressive optic atrophy from retinal ganglion cell death with an associated central visual field defect, decreased color vision and reduced contrast sensitivity. Prevalence of the disease is not well known but is estimated at 1 in 8,500-50,000 people worldwide (89). The most common mutations identified in mitochondrial genes include ND1 (G3460A), ND4 (G11778A), and ND6 (T14484C). These genes encode complex I (NADH dehydrogenase) of the electron transport chain necessary for mitochondrial energy production and cellular function (90). A G-to-A transition in mitochondrial DNA at position 11778 in the gene encoding subunit 4 of NADH dehydrogenase (ND4) leads to a position 340 arginine-tohistidine substitution that is responsible for approximately half of LHON cases.

Investigations into genetic therapy have been limited by the difficulty with delivering genes into the mitochondria of the ganglion cells. A strategy that has proved successful is allotopic expression (i.e. expression of the mitochondrial gene in the nucleus), optimized to target the mRNA to the outer surface of mitochondria. This facilitates translocation of the translated protein into mitochondria (91). Qi et al. showed that this approach would create the LHON phenotype in a normal mouse by delivering mutant 11778 human ND4 cDNA in an AAV2 vector to the mitochondria of retinal ganglion cells (92). This same technique was found to rescue a LHON rat model with intravitreal delivery of ND4 utilizing an AAV vector (93).

Currently, clinical trials are attempting to utilize allotopic expression to deliver ND4 in ganglion cells in patients with LHON G11778A mutations. Feuer et al. published the preliminary results for five patients that had been investigated following intravitreal injection of self-complementary AAV [scAAV]2(Y444,500,730F)-P1ND4v2. The first 3 participants were treated at the low dose of vector $\left(5 \times 10^{9} \mathrm{vg}\right)$, and the fourth participant was treated at the medium dose $\left(2.46 \times 10^{10} \mathrm{vg}\right)$. The fifth participant with visual loss for less than 12 months received the low dose. They reported no serious safety issues. An increase in VA was observed in two of the patients after gene delivery (NCT02161380) (94). However, the study design and outcomes reported in the paper have recently been called into question by some authors, with concerns about bias in the variable choice of baseline visit dates chosen for comparison, the likelihood of placebo effect due to one of the patients noticing an improvement on the first postoperative day and the possibility of spontaneous improvement of symptoms as part of the natural history of the disease in some patients (95).

Separately, Wan et al. released a report regarding a completed study from China that utilized a recombinant AAV2 carrying ND4 injected intravitreally in nine recruited 
patients (NCT01267422). These patients were followed over nine months and key findings included lack of systemic or ocular adverse events related to the vector, six of the nine patients measured a $0.3 \log$ MAR improvement in VA and these six patients had an associated visual field enlargement (96).

GenSight Biologics is sponsoring two phase 3 randomized double-masked shamcontrolled clinical trials ("RESCUE" and "REVERSE") of their GS010, AAV-mediated therapy containing the ND4 gene, for the treatment of LHON. The trials are to enroll 36 patients each and the first patient was recruited in February 2016, with the final patient to be enrolled expected in 2017. The primary endpoint is mean difference in BCVA in treated patients compared to sham patients. Topline results are expected at the end of the first quarter 2018 (97).

\subsection{Gene therapies for Achromatopsia:}

Achromatopsia (ACHM, rod monochromatism) is an autosomal recessive retinal condition that presents early in life with poor VA and color vision, photophobia, and nystagmus secondary to the absence of cone photoreceptors (98). It affects approximately 1 in 33,000 people in the US. Currently, six genes

(ATF6, CNGA3, CNGB3, GNAT2, PDE6C and PDE6H) have been linked to ACHM. Approximately 50\% carry mutations in the CNGB3 and 25\% carry mutations in the CNGA3 gene, which encode the two subunits of the cone cyclic nucleotide-gated (CNG) channel respectively (99). Other cases are associated with guanine nucleotide-binding protein G subunit alpha-2 (GNAT2) and phosphodiesterase 6C and 6H (PDE6C, $\mathrm{PDE} 6 \mathrm{H})$. All of these proteins play a critical role in cone phototransduction and their deficiency or malfunction results in retinal cone pathology (100). Mouse, canine, and sheep models have demonstrated the ability to replace these particular genes (101-103).

STZ eyetrial is sponsoring a dose escalation safety and efficacy phase I/II trial utilizing a single subretinal injection of rAAV.hCNGA3 for patients with CNGA3-linked achromatopsia (NCT02610582). MeiraGTx is also sponsoring a phase I/II trial to evaluate the safety for AAV-mediated CNGB3 delivery to patients with congenital ACHM and the company plans to initiate a similar phase I/II trial of AAV-mediated delivery of CNGA3 (NCT03001310). Applied Genetic Technologies Corporation (AGTC) is currently recruiting individuals with CNGB3 mutations into a phase I/II clinical trial to receive a subretinal injection of AAV expressing CNGB3 (rAAV2tYFPR1.7-hCNGB3) (NCT02599922), and is also sponsoring a dose escalation phase I/II clinical trial of AAV-mediated CNGA3 gene therapy in ACHM, with planned doses of $1.8 \times 10^{11} \mathrm{vg}, 4.5 \times 10^{11} \mathrm{vg}$, and $1.35 \times 10^{12} \mathrm{vg}$, and a primary endpoint of safety as well as secondary endpoints of VA and color vision (NCT02935517) (104).

\subsection{Gene therapies for X-Linked Retinoschisis (XLRS):}

XLRS is characterized by splitting of the retinal layers and the presence of intraretinal fluid-filled cysts, which results in a variable degree of visual dysfunction in young males. This separation leads to a characteristic decrease in the ERG b-wave with preservation of the a-wave secondary to the poor synaptic transmission between the photoreceptors and bipolar cells, resulting in the "negative ERG" (105). Estimates of the prevalence of $\underline{X-}$ 
linked retinoschisis vary from 1 in 5,000 to 1 in 25,000 (106). A mutation in retinoschisin (RS1), which plays a role in retinal organization and synapse structure, accounts for these findings. The absence of RS1 in the mouse model demonstrated similar findings to retinoschisis with fluid filled cavities and progressive photoreceptor cell death (107). Gene therapy has been underway to target this mutation in animal models (108). Unique to this condition, because of the weakness of the retina in retinoschisis, an approach with an intravitreal injection is favored over a subretinal approach (109). An AAV8-mediated delivery of RS1 induced reorganization of the photoreceptor and bipolar cell synapses with restoration of function in the RS1 knockout mouse model (110-111). This prompted the initiation of two clinical trials investigating the intravitreal delivery of AAV-RS1 vector in those affected by XLRS. AGTC is sponsoring a dose escalation phase I/II clinical trial of AAV-mediated RS1 gene therapy in XLRS, their lead program. Dosing will include $1 \times 10^{11} \mathrm{vg}, 3 \times 10^{11} \mathrm{vg}$, and $6 \times 10^{11} \mathrm{vg}$, with a primary endpoint of safety as well as secondary and supportive endpoints of VA, visual fields and ERG (NCT02416622) (112). The National Eye Institute is conducting an open-label of an AAV8-mediated RS1 gene therapy in XLRS (NCT02317887).

\section{CONCLUSION:}

The evolution of gene therapy has been remarkable over the last decade and has proven to be relatively safe in a multitude of small clinical trials, albeit with varying outcomes. With the optimization of viral vector transduction, improvements in cell-specific tropism, improved transgene expression through gene promoters, utilization of additional vectors with larger gene capacities, and advances in the surgical delivery of genetic treatments, the future of gene therapy shows great promise (113-114). Additionally, better understanding of the pathophysiology of retinopathies as well as the mechanisms that limit gene replacement therapy will facilitate further refinement of gene therapy for retinal degenerations.

\section{EXPERT OPINION:}

To date there is no approved treatment for the stabilization or reversal of vision loss in patients with IRDs. The rarity of these diseases limit large scale clinical trials. However, the partnership of industry sponsors with basic science researchers, incentivized by the Orphan Drug Act, have created a supportive environment for gene therapy in IRDs.

Gene therapy has an acceptable safety profile in many of the clinical trials, with low risk of local immunogenic response and no evidence of significant systemic adverse events. However, efficacy remains uncertain and variability exists in visual outcomes between disease processes, method of gene therapy and outcomes measured. In particular, traditional clinical trial endpoints involving VA, with its limited dynamic range and its predominant relationship to cone function, are problematic in advanced rod-mediated retinal degenerative disease or in children with dense amblyopia; consequently, alternative clinical trial endpoints have been developed. For instance, the results of the most recent LCA Phase 3 trials demonstrated an improvement in MLMT and FST, but the trend in improved VA did not reach statistical significance. However, MLMT may more comprehensively assess vision as it more functionally assesses VA, visual fields, 
light sensitivity, and mobility in these patients. Furthermore, these novel study endpoints are clinically meaningful to regulators.

In addition to efficacy endpoints, many issues and questions remain. For instance, even the nomenclature of various IRDs may require further development, given the overlap and variation among phenotypic diagnoses, in an era in which genotyping becomes more widely adopted, and gene-specific therapies are ultimately implemented. In addition, the safest and most effective approach for delivering the viral vector is unclear, especially as vector technology evolves. In order to minimize surgical complications, intravitreal delivery has been evaluated in XLRS and LHON, but this approach has the potential for lower penetration to the deeper retinal tissues, which are the main targets in many of the IRDs. Subretinal delivery likely fosters greater transduction over intravitreal delivery, but risks surgical complications during vector administration. Also, the longevity of gene expression from a one-time delivery of a vector genome is unknown and it is unclear if the eye can tolerate additional treatments to enhance cellular gene expression. Finally, adoption of gene therapy for IRDs will require widespread genetic screening to identify patients based on genotype, as well as new reimbursement models for one-time therapies.

In summary, gene therapy for IRDs is still in its early stages but holds great promise when treating monogenic IRDs, especially given the improvements in viral vector safety, more specific targeting to cell types/tissues, and improved surgical approaches for gene delivery.

\section{ARTICLE HIGHLIGHTS:}

- Gene therapy has a promising future in the treatment of degenerative retinal diseases.

- A variety of viral vectors and surgical delivery techniques are being investigated in clinical trials to identify the safest and most effective method for targeting pertinent retinal cellular structures.

- Local tolerability of vector therapy for IRDs has been demonstrated in numerous small studies and no serious systemic complications have been reported.

- Continued advancements in the understanding of the pathophysiology of IRDs will allow for improved, more targeted, genetic therapy.

- The monogenic nature of IRDs and lack of effective treatment to date makes gene therapy a compelling approach and continued investigation is warranted. 


\section{TABLE LEGEND:}

Table 1. Comparison of phase 1 Leber's Congenital Amaurosis safety report studies

Table 2: Ongoing or recently completed human gene therapy trials for inherited retinal and optic nerve degenerations

\section{FIGURE LEGEND:}

Figure 1: Subretinal injection: This figure demonstrates the location of a subretinal injection of therapeutic viral vectors, a procedure that is performed in an outpatient surgical setting. After pars plana vitrectomy to remove vitreous, a small retinotomy is created and the neurosensory retina is detached to create a small bleb. The vectors are then delivered directly into this iatrogenic space, between the photoreceptors and the retinal pigment epithelium. The volume and dose of viral vector, precise retinal location within the eye, and the number of retinotomies through which to deliver has yet to be been optimized. Reproduced figure with permission from Expert Opinion on Biological Therapy: Gene therapy for agerelated macular degeneration DOI: 10.1080/14712598.2017.1356817.

Figure 2: Intravitreal injection: This figure represents the location of an intravitreal injection of therapeutic viral vectors, a brief office-based procedure. Following local anesthesia and application of betadine to reduce the risk of infection, a smallgauge needle is inserted through the pars plana into the vitreous. The vector is injected directly into the vitreous humor and must traverse posteriorly through the vitreous and inner retinal layers in order to transduce the retinal pigment epithelium and photoreceptors. Reproduced figure with permission from Expert Opinion on Biological Therapy: Gene therapy for age-related macular degeneration DOI: 10.1080/14712598.2017.1356817. 


\section{REFERENCES:}

1. Sohocki MM, Daiger SP, Bowne SJ, et al. Prevalence of mutations causing retinitis pigmentosa and other inherited retinopathies. Human mutation. 2001;17(1):4251.

2. Sengillo JD, Justus S, Tsai YT, et al. Gene and cell-based therapies for inherited retinal disorders: An update. American journal of medical genetics Part C, Seminars in medical genetics. 2016;172(4):349-66.

3. Carter PJ and Samulski RJ. Adeno-associated viral vectors as gene delivery vehicles. Int J Mol Med. 2000;6(1):17-27.

4. Penaud-Budloo M, Le Guiner C, Nowrouzi A. Adeno-Associated Virus Vector Genomes Persist as Episomal Chromatin in Primate Muscle. J Virol. 2008; 82(16): 787585.

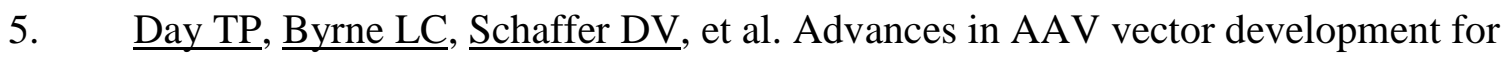
gene therapy in the retina. Adv Exp Med Biol. 2014;801:687-93.

6. Daya S and Berns KI. Gene therapy using adeno-associated virus vectors. Clin Microbiol Rev. 2008;21(4):583-93.

7. Surace EM and Auricchio A. Versatility of AAV vectors for retinal gene transfer. J Vis. 2008;48(3):353-59.

8. Hareendran S, Balakrishnan B, Sen D, et al. Adeno-associated virus (AAV) vectors in gene therapy: immune challenges and strategies to circumvent them. Rev Med Virol. 2013;23(6):399-413.

9. McCarty DM, Monahan PE, Samulski RJ. Self-complementary recombinant adeno-associated virus (scAAV) vectors promote efficient transduction independently of DNA synthesis. Gene Ther. 2001;8(16):1248-54.

10. McCarty DM. Self-complementary AAV vectors; advances and applications. Mol Ther. 2008;16(10):1648-56.

11. Everson EM and Trobirdge GD. Retroviral vector interactions with hematopoietic cells. Curr Opin Virol. 2016;21:41-6.

12. Sakuma T, Barry MA, Ikeda Y. Lentiviral vectors: basic to translational. Biochem J. 2012;443(3):603-18. 
13. Kumar M, Keller B, Makalou N, et al. Systematic Determination of the Packaging Limit of Lentiviral Vectors. Hum Gene Ther. 2004;12(15):1893-905.

14. Zufferey R, Dull T, Mandel RJ, et al. Self-inactivating lentivirus vector for safe and efficient in vivo gene delivery. J Virol. 1998;72:9873-80.

15. Streilein JW. Ocular immune privilege: therapeutic opportunities from an experiment of nature. Nat Rev Immunol. 2003;3(11):879-89.

16. Yu-Wai-Man P. Genetic manipulation for inherited neurodegenerative diseases: myth or reality? Br J Ophthalmol. 2016;100(10):1322-31.

17. Li Q, Miller R, Han PY, et al. Intraocular route of AAV2 vector administration defines humoral immune response and therapeutic potential. Mol Vis. 2008;14:1760-9. 18. Hanein S, Perrault I, Gerber S, et al. Leber congenital amaurosis: comprehensive survey of the genetic heterogeneity, refinement of the clinical definition, and genotypephenotype correlations as a strategy for molecular diagnosis. Hum Mutat. 2004;23(4):306-17.

19. den Hollander AI, Roepman R, Koenekoop RK, et al. Leber congenital amaurosis: genes, proteins and disease mechanisms. Prog Retin Eye Res. 2008;27(4):391-419.

20. Allikmets R. Leber congenital amaurosis: a genetic paradigm. Ophthalmic Genet. 2004;25(2):67-79.

21. Lorenz B, Gyurus P, Preising M, et al. Early-onset severe rod-cone dystrophy in young children with RPE65 mutations. Invest Ophthalmol Vis Sci. 2000;41(9):2735-42.

22. Thompson DA, Gyurus P, Fleischer LL, et al. Genetics and phenotypes of RPE65 mutations in inherited retinal degeneration. Invest Ophthalmol Vis Sci.

2000;41(13):4293-99.

23. Redmond TM, Yu S, Lee E, et al. Rpe65 is necessary for production of 11-cisvitamin A in the retinal visual cycle. Nat Genet. 1998;20(4):344-51.

24. Redmond TM, Poliakov E, Yu S, et al. Mutation of key residues of RPE65 abolishes its enzymatic role as isomerohydrolase in the visual cycle. Proc Natl Acad Sci USA. 2005;102(38):13658-63.

25. Acland GM, Aguirre GD, Ray J, et al. Gene therapy restores vision in a canine model of childhood blindness. Nat Genet. 2001;28(1):92-5.

26. Le Meur G, Stieger K, Smith AJ, et al. Restoration of vision in RPE65-deficient Briard dogs using an AAV serotype 4 vector that specifically targets the retinal pigmented epithelium. Gene Ther. 2007;14(4):292-303.

*27. Bainbridge JW, Smith AJ, Barker SS, et al. Effect of gene therapy on visual function in Leber's congenital amaurosis. New Engl J Med. 2008;358(21):2231-9.

* $\quad$ Earliest gene therapy clinical trial results for LCA

*28. Hauswirth WW, Aleman TS, Kaushal S, et al. Treatment of leber congenital amaurosis due to RPE65 mutations by ocular subretinal injection of adeno-associated virus gene vector: short-term results of a phase I trial. Hum Gene Ther. 2008;19(10):97990.

* $\quad$ Earliest gene therapy clinical trial results for LCA

*29. Maguire AM, Simonelli F, Pierce EA, et al. Safety and efficacy of gene transfer for Leber's congenital amaurosis. New Engl J Med. 2008;358(21):2240-8.

* $\quad$ Earliest gene therapy clinical trial results for LCA 
30. Maguire AM, High KA, Auricchio A, et al. Age-dependent effects of RPE65 gene therapy for Leber's congenital amaurosis: a phase 1 dose-escalation trial. Lancet. 2009;374(9701):1597-605.

31. Testa F, Maguire AM, Rossi S, et al. Three-year follow-up after unilateral subretinal delivery of adeno-associated virus in patients with Leber congenital Amaurosis type 2. Ophthalmology. 2013;120(6):1283-91.

32. Bainbridge JW, Mehat MS, Sundaram V, et al. Long-term effect of gene therapy on Leber's congenital amaurosis. New Engl J Med. 2015;372(20):1887-97.

33. Jacobson SG, Cideciyan AV, Roman AJ, et al. Improvement and decline in vision with gene therapy in childhood blindness. New Engl J Med. 2015;372(20):1920-6.

34. Bennett J, Wellman J, Marshall KA, et al. Safety and durability of effect of contralateral-eye administration of AAV2 gene therapy in patients with childhood-onset blindness caused by RPE65 mutations: a follow-on phase 1 trial. Lancet. 2016;388(10045):661-72.

35. Spark Therapeutics Announces New Positive Data from Continuation of Phase 3 Trial of Voretigene Neparvovec. 2016. Available from: http://ir.sparktx.com/phoenix.zhtml?c=253900\&p=irol-newsArticle\&ID=2194535

36. Spark Therapeutics Completes Rolling Biologics License Application Submission to FDA for Investigational Gene Therapy Voretigene Neparvovec. 2017. Available from: http://ir.sparktx.com/phoenix.zhtml?c=253900\&p=irol-newsArticle\&ID=2273905

37. Guylene Le Muer, Lebranchu P, Pereon Y, et al. Gene therapy in Leber congenital amaurosis due to RPE mutations: results of the first six patients included in a clinical trial. Invest Ophthalmol Vis Sci. 2013;54:5969.

38. Applied Gentic Technologies Corporation (AGTC). 2017. Available from: https://www.agtc.com/products

39. MeiraGTx. 2017. Available from: http://meiragtx.com/pipeline/

40. Boye SL, Peterson JJ, Choudhury S, et al. Gene Therapy Fully Restores Vision to the All-Cone Nrl(-/-) Gucy2e(-/-) Mouse Model of Leber Congenital Amaurosis-1. Hum Gene Ther. 2015;26(9):575-92.

41. Mihelec M, Pearson RA, Robbie SJ, et al. Long-term preservation of cones and improvement in visual function following gene therapy in a mouse model of leber congenital amaurosis caused by guanylate cyclase-1 deficiency. Hum Gene Ther. 2011;22(10):1179-90.

42. Pawlyk BS, Smith AJ, Buch PK, et al. Gene replacement therapy rescues photoreceptor degeneration in a murine model of Leber congenital amaurosis lacking RPGRIP. Invest Ophthalmol Vis Sci. 2005;46(9):3039-45.

43. Sanchez-Alcudia R, Garcia-Hoyos M, Lopez-Martinez MA, et al. A Comprehensive Analysis of Choroideremia: From Genetic Characterization to Clinical Practice. PLoS One. 2016;11(4):e0151943.

44. MacLaren RE, Groppe M, Barnard AR, et al. Retinal gene therapy in patients with choroideremia: initial findings from a phase 1/2 clinical trial. Lancet. 2014;383(9923):1129-37.

45. Preising $\mathrm{M}$ and Ayuso C. Rab escort protein 1 (REP1) in intracellular traffic: a functional and pathophysiological overview. Ophthalmic Genet. 2004;25(2):101-10. 
*46. Dimopoulos IS, Chan S, MacLaren RE, et al. Pathogenic mechanisms and the prospect of gene therapy for choroideremia. Expert Opin Orphan Drugs. 2015;3(7):787-98.

* Great article reviewing the complex pathophysiology of choroideremia.

47. Alory $\mathrm{C}$ and Balch WE. Organization of the Rab-GDI/CHM superfamily: the functional basis for choroideremia disease. Traffic. 2001;2(8):532-43.

48. Lukashev AN, Zamyatnin AA. Viral Vectors for Gene Therapy: Current State and Clinical Perspectives. Biochemistry. 2016;81(7):700-8.

49. Zinkernagel MS and MacLaren RE. Recent advances and future prospects in choroideremia. Clin Ophthalmol. 2015;9:2195-200.

50. Bennett J, Maguire AM, Cideciyan AV, et al. Stable transgene expression in rod photoreceptors after recombinant adeno-associated virus-mediated gene transfer to monkey retina. Proc Natl Acad Sci USA. 1999;96(17):9920-5.

51. Tolmachova T, Tolmachov OE, Barnard AR, et al. Functional expression of Rab escort protein 1 following AAV2-mediated gene delivery in the retina of choroideremia mice and human cells ex vivo. J Mol Med. 2013;91(7):825-37.

52. Rosser DA, Cousens SN, Murdoch IE. How sensitive to clinical change are

ETDRS logMAR visual acuity measurements? Invest Ophthalmol Vis Sci. 2003;44(8):3278-81.

53. Edwards TL, Jolly JK, Groppe M, et al. Visual Acuity after Retinal Gene Therapy for Choroideremia. New Engl J Med. 2016;374(20):1996-8.

54. NightstaRx. 2017. Available from: https://www.nightstartx.com/our-programs/

55. Spark Therapeutics. SPK-7001: Choroideremia. 2017. Available from: http://sparktx.com/scientific-platform-programs/

56. Tolmachova T, Wavre-Shapton ST, Barnard AR, et al. Retinal pigment epithelium defects accelerate photoreceptor degeneration in cell type-specific knockout mouse models of choroideremia. Invest Ophthalmol Vis Sci. 2010;51(10):4913-20.

57. Syed N, Smith JE, John SK, et al. Evaluation of retinal photoreceptors and pigment epithelium in a female carrier of choroideremia. Ophthalmology. 2001;108(4):711-20.

58. MacDonald IM, Chan CC, Hiriyanna KT, et al. REP-1 localization in the eye. Invest Ophthalmol Vis Sci. 2005;46 ARVO abstract 540 .

59. Black A, Vasireddy V, Chung DC, et al. Adeno-associated virus 8-mediated gene therapy for choroideremia: preclinical studies in in vitro and in vivo models. J Gene Med. 2014;16(5-6):122-30.

60. Vandenberghe LH, Bell P, Maguire AM, et al. Dosage thresholds for AAV2 and AAV8 photoreceptor gene therapy in monkey. Sci Transl Med. 2011;3(88):88ra54.

61. Hartong DT, Berson EL, Dryja TP. Retinitis pigmentosa. Lancet. 2006;368(9549):1795-809.

62. Mackay DS, Henderson RH, Sergouniotis PI, et al. Novel mutations in MERTK associated with childhood onset rod-cone dystrophy. Mol Vis. 2010;16:369-77.

63. Shahzadi A, Riazuddin SA, Ali S, et al. Nonsense mutation in MERTK causes autosomal recessive retinitis pigmentosa in a consanguineous Pakistani family. The $\mathrm{Br} \mathrm{J}$ Ophthalmol. 2010;94(8):1094-9. 
64. Ostergaard E, Duno M, Batbayli M, et al. A novel MERTK deletion is a common founder mutation in the Faroe Islands and is responsible for a high proportion of retinitis pigmentosa cases. Mol Vis. 2011;17:1485-92.

65. D'Cruz PM, Yasumura D, Weir J, et al. Mutation of the receptor tyrosine kinase gene Mertk in the retinal dystrophic RCS rat. Hum Mol Genet. 2000;9(4):645-51. 66. Smith AJ, Schlichtenbrede FC, Tschernutter M, et al. AAV-Mediated gene transfer slows photoreceptor loss in the RCS rat model of retinitis pigmentosa. Mol Ther. 2003;8(2):188-95.

67. Conlon TJ, Deng WT, Erger K, et al. Preclinical potency and safety studies of an AAV2-mediated gene therapy vector for the treatment of MERTK associated retinitis pigmentosa. Hum Gene Ther Clin Dev. 2013;24(1):23-8.

68. Ghazi NG, Abboud EB, Nowilaty SR, et al. Treatment of retinitis pigmentosa due to MERTK mutations by ocular subretinal injection of adeno-associated virus gene vector: results of a phase I trial. Hum Genet. 2016;135(3):327-43.

69. Pawlyk BS, Bulgakov OV, Sun X, et al. Photoreceptor rescue by an abbreviated human RPGR gene in a murine model of X-linked retinitis pigmentosa. Gene Ther. 2016;23(2):196-204.

70. Beltran WA, Cideciyan AV, Lewin AS, et al. Gene therapy rescues photoreceptor blindness in dogs and paves the way for treating human X-linked retinitis pigmentosa. Proc Natl Acad Sci USA. 2012;109:2132-7.

**71. Farrar GJ, Millington-Ward S, Chadderton N, et al. Gene-based therapies for dominantly inherited retinopathies. Gene Ther. 2012;19(2):137-44.

** Methods to overcome the challenges of treating dominant mutations with gene therapy.

72. Palfi A, Chadderton N, O'Reilly M, et al. Efficient gene delivery to photoreceptors using AAV2/rh10 and rescue of the Rho(-/-) mouse. Mol Ther Methods Clin Dev. 2015;2:15016.

73. Millington-Ward S, Chadderton N, O'Reilly M, et al. Suppression and replacement gene therapy for autosomal dominant disease in a murine model of dominant retinitis pigmentosa. Mol Ther. 2011;19(4):642-9.

74. Mathur P, Yang J. Usher syndrome: Hearing loss, retinal degeneration and associated abnormalities. Biochim Biophys Acta. 2015;1852(3):406-20.

75. Lentz J and Keats B. Usher Syndrome Type I. GeneReviews. 2016. Available from: https://www.ncbi.nlm.nih.gov/books/NBK1265/

76. Hasson T, Heintzelman MB, Santos-Sacchi J, et al. Expression in cochlea and retina of myosin VIIa, the gene product defective in Usher syndrome type 1B. Proc Natl Acad Sci USA. 1995;92(21):9815-9.

77. Hashimoto T, Gibbs D, Lillo C, et al. Lentiviral gene replacement therapy of retinas in a mouse model for Usher syndrome type 1B. Gene Ther. 2007;14(7):584-94.

78. Oxford BioMedica and Sanofi-Aventis Enter New Collaboration to Develop Gene-Based Treatments for Ocular Diseases. 2009. Available from: http://www.oxfordbiomedica.co.uk/pipeline.

79. Fink TL, Klepcyk PJ, Oette SM, et al. Plasmid size up to $20 \mathrm{kbp}$ does not limit effective in vivo lung gene transfer using compacted DNA nanoparticles. Gene Ther. 2006;13(13):1048-51. 
80. Konstan MW, Davis PB, Wagener JS, et al. Compacted DNA nanoparticles administered to the nasal mucosa of cystic fibrosis subjects are safe and demonstrate partial to complete cystic fibrosis transmembrane regulator reconstitution. Hum Gene Ther. 2004;15(12):1255-69.

81. Han Z, Conley SM, Makkia R, et al. Comparative analysis of DNA nanoparticles and AAVs for ocular gene delivery. PloS one. 2012;7(12):e52189.

82. Blacharski PA. Fundus flavimaculatus. New York: Raven Press. 1988:135-9.

83. Petit L, Punzo C. Gene therapy approaches for the treatment of retinal disorders. Discov Med. 2016;22(121):221-9.

84. Tsybovsky Y, Molday RS, Palczewski K. The ATP-binding cassette transporter ABCA4: structural and functional properties and role in retinal disease. Adv Exp Med Biol. 2010;703:105-25.

85. Han Z, Conley SM, Naash MI. Gene therapy for Stargardt disease associated with ABCA4 gene. Adv Exp Med Biol. 2014;801:719-24.

86. Kong J, Kim SR, Binley K, et al. Correction of the disease phenotype in the mouse model of Stargardt disease by lentiviral gene therapy. Gene Ther. 2008;15(19):1311-20.

87. Lu LJ, Liu J, Adelman RA. Novel therapeutics for Stargardt disease. Graefe's Arch Clin Exp Ophthalmol. 2017;251(10):2299-309.

88. Binley K, Widdowson P, Loader J, et al. Transduction of photoreceptors with equine infectious anemia virus lentiviral vectors: safety and biodistribution of StarGen for Stargardt disease. Invest Ophthalmol Vis Sci. 2013;54(6):4061-71.

89. Yu-Wai-Man P and Chinnery PF. Leber Hereditary Optic Neuropathy.

GeneReviews. 2000. Available from: https://www.ncbi.nlm.nih.gov/books/NBK1174/.

90. Cwerman-Thibault H, Augustin S, Ellouze S, et al. Gene therapy for mitochondrial diseases: Leber Hereditary Optic Neuropathy as the first candidate for a clinical trial. C R Biol. 2014;337(3):193-206.

91. Bonnet C AS, Ellouze S, Bénit P, et al. The optimized allotopic expression of ND1 or ND4 genes restores respiratory chain complex I activity in fibroblasts harboring mutations in these genes. Biochim Biophys Acta. 2008;1783(10):1707-17.

92. Qi X, Sun L, Lewin AS, et al. The mutant human ND4 subunit of complex I induces optic neuropathy in the mouse. Invest Ophthalmol Vis Sci. 2007;48(1):1-10. **93. Ellouze S, Augustin S, Bouaita A, et al. Optimized allotopic expression of the human mitochondrial ND4 prevents blindness in a rat model of mitochondrial dysfunction. Am J Hum Genet. 2008;83(3):373-87.

** Interesting approach to trageting the mitochondria of retinal ganglion cells. 94. Feuer WJ, Schiffman JC, Davis JL, et al. Gene Therapy for Leber Hereditary Optic Neuropathy: Initial Results. Ophthalmology. 2016;123(3):558-70.

95. Dimopoulos IS, Xu M. Re: Feuer et al.: Gene therapy for Leber hereditary optic neuropathy: initial results (Ophthalmology 2016;123:558-570). Ophthalmology. 2017;124(3):e22.

96. Wan X, Pei H, Zhao MJ, et al. Efficacy and Safety of rAAV2-ND4 Treatment for Leber's Hereditary Optic Neuropathy. Sci Rep. 2016;6:21587.

97. GenSight. GS010. 2017. Available from: http://www.gensightbiologics.com/index.php?page=lhon-leber-hereditary-optic-neuropathy 
98. Zobor D, Zobor G, Kohl S. Achromatopsia: on the doorstep of a possible therapy. Ophthalmic research. 2015;54(2):103-8.

99. Michalakis S, Schon C, Becirovic E, Biel M. Gene Therapy for Achromatopsia. J Gene Med. 2017;19(3): DOI 10.1002/jgm.2944.

100. Kohl S and Hamel C. Clinical utility gene card for: Achromatopsia - update 2013. Eur J Hum Genet. 2013;21(11): DOI 10.1038/ejhg.2013.44.

101. Alexander JJ, Umino Y, Everhart D, et al. Restoration of cone vision in a mouse model of achromatopsia. Nat Med. 2007;13(6):685-7.

102. Komaromy AM, Alexander JJ, Rowlan JS, et al. Gene therapy rescues cone function in congenital achromatopsia. Hum Mol Genet. 2010;19(13):2581-93.

103. Pang JJ, Deng WT, Dai X, et al. AAV-mediated cone rescue in a naturally occurring mouse model of CNGA3-achromatopsia. PloS one. 2012;7(4):e35250.

104. Applied Gentic Technologies Corporation. Achromatopsia. 2017. Available from: https://www.agtc.com/products/achromatopsia

105. Schubert T, Wissinger B. Restoration of synaptic function in sight for degenerative retinal disease. J Clin Invest. 2015;125(7):2572-5.

106. Molday RS, Kellner U, Weber B. X -linked juvenile retinoschisis: Clinical diagnosis, genetic analysis, and molecular mechanisms. Prog Retin Eye Res. 2012;31(3):195-212.

107. Weber BH, Schrewe H, Molday LL, et al. Inactivation of the murine X-linked juvenile retinoschisis gene, Rs1h, suggests a role of retinoschisin in retinal cell layer organization and synaptic structure. Proc Natl Acad Sci USA. 2002;99(9):6222-7. 108. Molday LL, Min SH, Seeliger MW, et al. Disease mechanisms and gene therapy in a mouse model for X-linked retinoschisis. Adv Exp Med Biol. 2006;572:283-89. 109. Byrne LC, Ozturk BE, Lee T, et al. Retinoschisin gene therapy in photoreceptors, Muller glia or all retinal cells in the Rs1h-/- mouse. Gene Ther. 2014;21(6):585-92.

110. Ou J, Vijayasarathy C, Ziccardi L, et al. Synaptic pathology and therapeutic repair in adult retinoschisis mouse by AAV-RS1 transfer. J Clin Invest. 2015;125(7):2891-903.

111. Ye GJ, Conlon T, Erger K, Sonnentag P, et al. Safety and Biodistribution Evaluation of rAAV2tYF-CB-hRS1, a Recombinant Adeno-Associated Virus Vector Expressing Retinoschisin, in RS1-Deficient Mice. Hum Gene Ther Clin Dev. 2015;26(3):177-84.

112. Applied Gentic Technologies Corporation. X Linked Retinoschisis. 2017. Available from: https://www.agtc.com/products/x-Linked-retinoschisis 113. Gao G VL, Wilson JM. New recombinant serotypes of AAV vectors. Curr Gene Ther. 2005;5(3):285-97.

114. Mitchell AM NS, Warischalk JK, Samulski RJ. AAV's anatomy: roadmap for optimizing vectors for translational success. Curr Gene Ther. 2010;10(5):319-40. 